\title{
Contents, Vol. 106, 1943
}

\section{Index}

Bangerter, A., Pterygiumoperation und Deckung von Bindehautdefekten 316

Bischler, Vera, Malformations de Гæil et malformations du

cæur 169

Blok, C. J., Augensalben und ihr therapeutischer Effekt . 57

Blum, J.-D., Indications et résultats de la corépraxie deFranceschetti. (Avec un aperçu historique des operations de pupille artificielle.) 1, 66

Bruckner, R., Auge und Cholinesterase. 2. Mitteilung. Vor-kommen und Verteilung von

Cholinesterase in derUvea 200

Bürki, E., Schmíd, A., und Saubermann, G., Erfahrungen

mit Cibazol in der Ophthalmologie 113

Cseh, E., vide Szabó, G.

Delia Casa, F., Ein Adaptometer für den praktischen Arzt 143, 189

Kiss, F., Der Blutkreislauf des Auges 225

Krayenbühl, H., und Schmíd, A. E., Zur Lokalisation intra-kranieller-orbitaler Dermoide. Beitrag zur Differential-diagnose des unilateralen Exophthalmus . . . 251

Longhena, L., Beitrag zum Studium der retroretinalen Flüs-

sigkeit bei Netzhautablösung 27, 80

Radnót, Magda, Hypophysenvorderlappen und der intra-

okulare Druck 182

- $\quad$ Ein seltener Fall von Linsenluxation .... 312

Saubermann, G., vide Bürki, E.

Schmid, A. E., Distribution de Tlrgamid dans les tissus et

les liquides oculaires 289

vide Bürki, E.

vide Krayenbühl, $\mathrm{H}$.

Süllmann, H., Ueber den glykolytischen Abbau von Glucose

und Fructose durch Netzhautgewebe ... . 301

Szabó, G., und Cseh, E., Sklera-Neurinom in der Nähe des

Limbus

14

Zeeman, W. P. C, Zwei Fälle von Glaucoma phacogeneticum mit anatomischem Befund $\quad 136$

- Binasale Hemianopsie und Akromegalie . . . 271

UEBERSICHTSREFERATE - REVIEWS - REVUES

Babel, J., Uvée. (Années 1939 à 1941) 41

GESELLSCHAFTSBERICHTE - SOCIETY TRANSACTIONS - SOCIÉTÉS

Sitzungsberichit $\mathrm{d}^{1} / 8 \mathrm{r} 105$. Ve $\tau$ sammilung der Niederläraddischen Opbẃall •

miøliogiischen Geseliischaft in Amsterdam am 12./1S. Dezember 194293 
Sitzungsibericht der 106. Vers < amn $\tau$ luung der Niederländ'ischen Ophthalmologischen Geseilílschaft in Leiden am G. Jurai 1943 . . . 321

Gro ·pe ophitalimioil·ogique $\mathrm{d}^{1} / 8$ Lémian. Resume des communications d'après auto-référés par John-D. Blum (Geneve):

Reunion du ler mars 1942 à ГHôpitaГ ophtalmique1 d > e Lausanne

Reunion du 14 février 1943 à la Clinique ophtalmologique uni-

versitaire de Geneve

161

Reunion du 4 avГÜ1 1943 à 1'Hôpital ophtalmique de Lausanne. 166

Veneindgung dier Basíler Autge·nårzte. Sitzun³/4 vom 13. Mai 1943 in der

Uniiversitäts-Augenkli $\pi$ ik Basel 213

NECROLOGIA Marcel Danis $\dagger \quad 110$

$\Lambda$ uguste Dufour $\dagger \quad$ Ill

B. BUCHBESPRECHUNGEN - BOOKS REVIEW - LIVRES NOUVEAUX

Ill, 223 